\title{
GAIA Level 2 First Trimester Spontaneous Abortion
}

National Cancer Institute

\section{Source}

National Cancer Institute. GAIA Level 2 First Trimester Spontaneous Abortion. NCI

Thesaurus. Code C128777.

GAIA Level 2 First Trimester Spontaneous Abortion is defined by three criteria: first, it does not qualify as a Level 1 First T rimester Spontaneous Abortion; second, at least one of the following three requirements must be met: a) Crown-rump length greater than 7 $\mathrm{mm}$ and no visible heartbeat, confirmed by transvaginal ultrasound; OR b) Crown-rump length greater than $15 \mathrm{~mm}$ and no visible heartbeat, confirmed by abdominal ultrasound; $\mathrm{OR}$ c) Mean gestational sac diameter is $25 \mathrm{~mm}$ or more and no visible embryo; third, gestational age is within the pre-defined range for the selected abortion definition as assessed by maternal and/or fetal parameters (Level 1-2) (using Brighton Preterm Birth Gestational Age algorithm). 